\title{
Perception of emotion from moving body cues in photographs
}

\author{
KATHY L. WALTERS and RICHARD D. WALK \\ George Washington University, Washington, D.C.
}

\begin{abstract}
To investigate whether the dynamic moving condition in a videotape would transfer to a photograph, we showed subjects still photographs of emotions (anger, disgust, fear, happiness, sadness, and surprise) depicted as points of light in dynamic light displays. Overall recognition was $70.4 \%$ with the dynamic light displays, but it dropped to $25.5 \%$ with the blurred tracks in the photographs, significantly above a chance level of $16.7 \%$, but not large. Happiness, anger, and sadness were the most accurately recognized. The transfer effect of abstract movement to a still photograph may not be high, but the most active emotions (happiness, anger) and the most inactive one (sadness) do transfer. Our results may have some implications for the artistic rendition of movement in pictures.
\end{abstract}

When points of light are placed on the joints of the body and the illumination dimmed so that an observer sees only the dots of light in motion, the observer sees the points of light as a person moving. Without movement, the observer simply sees an abstract light pattern without meaning. Johansson (1973), the inventor of the technique, showed that the time for recognition is very short-about a quarter of a second-but that movement is necessary.

Suppose the movement were transferred to a still photograph. One would observe the tracks made by the moving lights, but one could not observe the actual movement.

The problem with this transferal is related to the problem of obtaining dynamic activity from a still picture. In discussing movement, Arnheim (1974) commented that the wish of the dancer is to project "a pattern of visual forces ... whose impact is immediately felt" (p. 409). But a moving dancer is not a picture of one, and Arnheim discussed the difficulty of getting an impression of movement from a photograph. The artist or photographer cannot rely on the past experience of the observer, but must try to use visual forces in the picture itself. One method is to use oblique lines or forms, and postural deviations are a common method of depicting movement (Friedman \& Stevenson, 1980). Blurred lines are occasionally used to indicate movement in pictures, combined with a rendering of the figure itself (Friedman \& Stevenson, 1980).

Our method uses the blurred lines alone to investigate whether subjects can distinguish the meaning of patterns of movement during sequences of emotional movement. Prior research (Walk \& Homan, 1984; Walk, Walters, $\&$ Samuel, 1985) has shown that the dynamic light patterns of a person depicting emotions are as well recognized as are facial expressions of emotion. The best recog-

\footnotetext{
This research was supported in part by NIH Biomedical Research Support Grant 2-S07-RR07019 to George Washington University. Requests for reprints should be sent to Richard D. Walk, Department of Psychology, George Washington University, Washington, DC 20052.
}

nition was for lights on all 12 of the major joints of the body $(84 \%)$, somewhat less for the 6 illumination points on the upper torso (69\%), and still less for the 6 points on the lower torso (57\%) (Walk et al., 1985).

With the recognition of emotions from the moving lights as a base, we decided to determine how well subjects can recognize emotions in tracks or streaks of light in still photographs. We varied the placement of the light cues, using the points of light on the joints of the entire body, of the upper half, and of the lower half. In addition, we asked subjects to distinguish between a walking and a dancing figure in a photograph, and we investigated whether the sex of the observer influenced correct identification.

\section{METHOD}

\section{Subjects}

The subjects were 66 students in an undergraduate course in experimental psychology. There were 47 females and 19 males from five different laboratory sections.

\section{Stimulus Materials}

The Kodachrome slides were prepared by photographing a videotaped image displayed on a black-and-white television monitor. The videotape was of a performer dressed in black clothing with a hood over the head. White cotton balls, $4.0 \mathrm{~cm}$ in diameter, were taped to the clothing at the joints. The performer portrayed six emotions-anger, disgust, fear, happiness, sadness, and surprise-against a black background. In the full-cue condition, cotton balls were placed on the joints of the shoulders, elbows, wrists, hips, knees, and ankles (12 cues). In the upper and lower conditions, using only 6 cues, the placements were at the shoulders, elbows, and wrists (upper), and the hips, knees, and ankles (lower). The videotape was played on a Sony Betamax connected to a $30-\mathrm{cm}$ black-and-white Sony monitor. When the contrast on the monitor was at a maximum, only the cotton balls were seen. The slides were made by photographing the video display (used by Walk et al., 1985) with Kodachrome 64 film at f5.6 for $1 \mathrm{sec}$ in a dark room. Twenty-six slides were prepared. Each of the six emotions was depicted once in the upper and lower torso conditions and twice in the full-cue condition. In addition, two slides were made by photographing the videotape display while the performer walked and danced. (See Figure 1 for the full-cue and the walking and dancing slides.) 

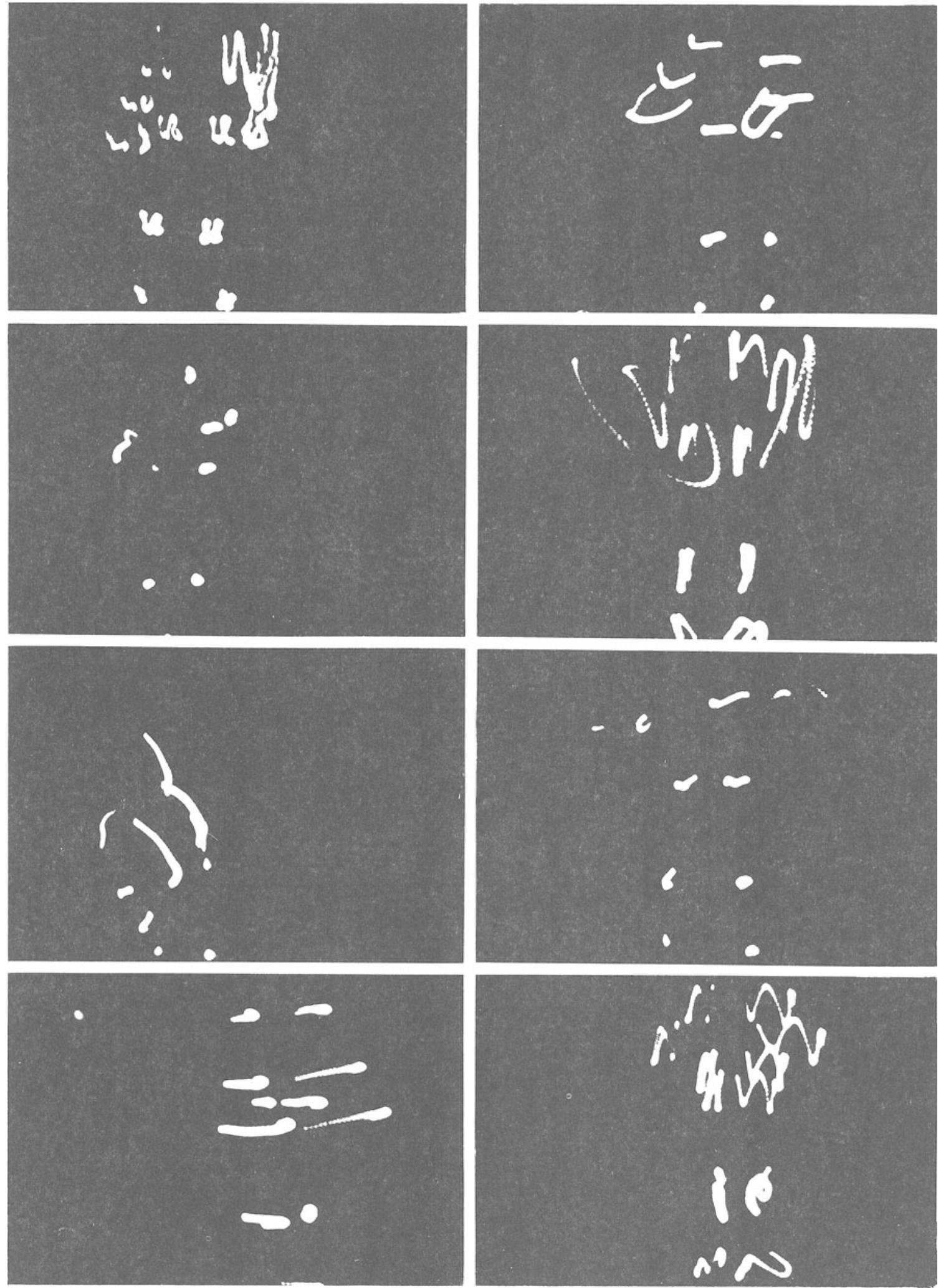

Figure 1. The full-cue stimulus photographs used in the experiment. Reading left to right and top to bottom, they are anger and disgust (first row), fear and happiness (second row), sadness and surprise (third row), walking and dancing (bottom row). 


\section{Procedure}

The subjects were informed that they would be shown four blocks of six slides depicting emotions. The subjects were then given response sheets containing blanks for each trial and a list of the six emotions. The subjects were shown the slides in four blocks of six trials. The two blocks of full-cue condition slides were shown successively. Each laboratory section saw a different order of the cue conditions (e.g., upper, lower, full, full), as well as a different emotion order within the cue condition. Three of the laboratory sections saw the walking slide first, and two sections saw the dancing slide first. The slides were projected for approximately $20 \mathrm{sec}$ each, and $20 \mathrm{sec}$ were allowed between blocks of trials. After the emotions were displayed, the subjects were told that they would be shown two additional slides, one portraying dancing and one walking. They were to identify each slide as either "dance" or "walk."

\section{RESULTS}

A repeated measures analysis of variance (ANOVA) revealed that placement of the cues (full, upper, lower) was not significant. More specifically, the average correct recognition was $27 \%$ for full cues, $24 \%$ for upper cues, and $26 \%$ for lower cues, a nonsignificant difference for type of cue. The second set of full-cue data was therefore dropped from all analyses, and an ANOVA was conducted comparing emotion and sex of subject.

An unweighted-means repeated measure ANOVA indicated that only type of emotion was significant $[F(5,320)$ $=3.13, p<.01]$. The gender of the subject was not significant as a variable and neither was the sex $\times$ emotion interaction. The Newman-Keuls test showed that recognition of happiness and anger was significantly superior to recognition of disgust $(p<.01$ and $p<.05$, respectively) but not significantly different from each other. Recognition of happiness was also significantly superior to recognition of fear and surprise $(p<.05)$.

Table 1 illustrates the percentages of the emotions identified correctly and also the labels attached by the subjects to each emotional depiction. Disgust was the only emotion labeled at a chance level (16.67\%). Table 1 also shows some confusions that were difficult to interpret. Sadness and fear are inactive emotions, and it may be appropriate for them to be confused with each other. Sur-

Table 1

Percentages of Emotions Identified for Each Photograph (All Cue Types)

\begin{tabular}{lrrrccc}
\hline & \multicolumn{6}{c}{ Photograph Presented } \\
\cline { 2 - 7 } Response & Happiness & Anger & Sadness & Surprise & Fear & Disgust \\
\hline Happiness & $\mathbf{3 5 . 3 5}$ & 15.15 & 7.07 & 13.64 & 11.11 & 15.15 \\
Anger & 14.65 & $\mathbf{2 9 . 2 9}$ & 9.09 & 10.61 & 11.11 & 18.69 \\
Sadness & 8.08 & 4.55 & $\mathbf{2 6 . 7 7}$ & 18.69 & 20.71 & 15.15 \\
Surprise & 17.17 & 17.17 & 8.08 & $\mathbf{2 2 . 7 3}$ & 12.12 & 20.20 \\
Fear & 8.59 & 14.65 & 25.76 & 18.69 & $\mathbf{2 2 . 2 2}$ & 10.10 \\
Disgust & 12.63 & 13.64 & 18.69 & 13.13 & 19.70 & $\mathbf{1 6 . 6 7}$ \\
\hline
\end{tabular}

prise was confused with sadness and fear, and disgust was identified as anger and surprise more than it was correctly identified.

Eighty-seven percent of the subjects identified the walking and the dancing slides correctly.

\section{DISCUSSION}

Even when the moving points of light of event perception are reduced to streaks of light on a photograph, emotion can be identified somewhat accurately. As Walk and Homan (1984) and Ekman and Friesen (1971) found, happiness was identified more accurately than the other emotions. In agreement with Walk and Homan (1984), our study showed that anger was identified second best. But the streaks on the photographs cannot be substituted for the actual emotional depiction in motion, as indicated by the results of Walk et al.'s (1985) study: the recognition of happiness with the event perception of moving dots was almost perfect in their study ( $96 \%$ average of all cues) but was only $35 \%$ in this study, and recognition of anger was very high in Walk et al.'s study (66\% overall, but $94 \%$ with full cues) but was only $29 \%$ in this study.

The best way to look at these data is in terms of the activity revealed in the streaks of light. One can identify a happy woman by "the bounce in her step," and anger may be recognizable because of physically violent movements. Darwin (1872/1955) referred to sadness as "silent, motionless grief" and sadness, an inactive body emotion, was our third best identified depiction. The ambiguity of fear, surprise, and disgust on the activity scale may reflect the difficulty of translating them into the streaks of light, although, as event perception, they are easily identified.

The dynamic visual forces revealed by our technique come from the actual movement of the human figure and have a certain intrinsic aesthetic beauty. Another procedure, such as a sorting method in which the subject compares the photographs simultaneously rather than successively, might be better for appreciating the meaning of these dynamic forces.

\section{REFERENCES}

Arnheim, R. (1974). Art and visual perception (rev. ed.). Berkeley: University of California Press.

DARWIN, C. (1955). Expression of the emotions in man and animal. New York: Philosophical Library. (Original work published 1872)

Ekman, P., \& Friesen, W. V. (1971). Constants across cultures in the face and emotion. Journal of Personality \& Social Psychology, 17, 124-129.

Friedman, S. L., \& Stevenson, M. B. (1980). Perception of movement in pictures. In M. A. Hagen (Ed.), The perception of pictures. Vol. 1. Alberti's window: The projective model of pictorial information (pp. 225-255). New York: Academic Press.

Johansson, G. (1973). Visual perception of biological motion and a model for its analysis. Perception \& Psychophysics, 14, 201-211.

WALK, R. D., \& Homan, C. P. (1984). Emotion and dance in dynamic light displays. Bulletin of the Psychonomic Society, 22, 437-440.

Walk, R. D., Walters, K. L., \& Samuel, J. M. F. (1985, March). Event perception of emotion as a function of placement of cues on the body. Paper presented at the meeting of the Eastern Psychological Association, Boston.

(Manuscript received for publication August 12, 1987.) 